\title{
Role of Dielectric Property of Blood in Detection of Renal Failure
}

\author{
Arpita Gupta*1 ${ }^{1}$ G.U.Kharat ${ }^{2}$ \\ ${ }^{1}$ EC dept, KEC, Ghaziabad, 201006, India \\ ${ }^{2}$ EC dept, Sharadchandra Pawar College of Engineering, Pune, 412409, India \\ Corresponding Author \\ Arpita Gupta \\ EC dept, \\ KEC, Ghaziabad, 201006 \\ India \\ Phone No. 91-9891989944 \\ Fax No. 0120-2898513 \\ Email: arpita_gupta15@yahoo.co.in
}

Received: 15 May 2015; | Revised: 10 August 2015; | Accepted: 29 August 2015

\begin{abstract}
The dielectric property of blood cell is of great significance and has been attracting the attention of many researchers since decades. Renal failure or kidney failure is a very common problem now a days and early diagnosis can help these patients in their treatment. Creatinine is the waste product of human body which is generated due to muscle metabolisms. One of the functions of kidney is to maintain the creatinine level in the body. Creatinine level is an indicator of functioning of kidney. The creatinine content of blood of a subject changes the dielectric property of blood. The authors have correlated the dielectric property of blood with the creatinine level. It was observed that the pre dialysis blood where the creatinine level is more, the dielectric of the sample is less than that of post dialysis sample where the creatinine level is less. Thus by measuring the dielectric property of the blood, the creatinine value can be estimated which is a clear indication of functioning of kidney. The authors believe that the dielectric property of blood cell can effectively help in diagnosis of renal diseases. The paper clearly shows how dielectric property of blood can be helpful in detecting the malfunctioning of kidney. Also the capacitance of the blood cell depends on dielectric property of blood. In this paper, a relation between blood capacitance and creatinine has been developed. Blood capacitance which can be easily measured can approximately predict the value of blood creatinine which helps in diagnosis and treatment of patients with renal disorder. The collected data is analyzed statistically in MINITAB software. The software is very useful for process improvement with Monte Carlo simulation.
\end{abstract}

Keywords: Dielectric property of blood, renal failure, creatinine, Kidney, blood capacitance, MINITAB 


\section{Introduction}

The kidneys are responsible for the homeostasis of the blood. They prevent the buildup of wastes and extra fluid in the body and keep the levels of electrolytes stable in the body such as level of sodium, potassium, and phosphate. They also make hormones that help in the following ways:-

\section{$\checkmark$ Regulating blood pressure \\ $\checkmark$ Make red blood cells \\ $\checkmark$ Bones stay strong}

The kidneys also maintain the blood creatinine in a normal range [1]. Creatinine is a waste product in human blood that is a result of muscle metabolism. It is normally removed from blood by kidneys. Normal range of creatinine in the blood is approximately 0.6 to 1.2 milligrams $(\mathrm{mg})$ per deciliter $(\mathrm{dL})$ in male adults and 0.5 to 1.1 milligrams per deciliter in female adults. But when kidney function slows down, the creatinine level rises [2]. Creatinine has been found to be a fairly reliable indicator of kidney function [3]. Increased value of creatinine level signifies impaired kidney function or kidney disease. As the kidneys become impaired for any reason, the creatinine level in the blood rises due to poor cleaning of creatinine by the kidneys. Abnormally high levels of creatinine thus warn of possible malfunction or failure of the kidneys $[4,5]$. It is for this reason that standard blood tests routinely check the amount of creatinine in the blood. Dialysis is a process by which the blood is cleaned by an artificial kidney. The creatinine values of pre and post dialysis blood samples are different [6]. Obviously the creatinine value of blood samples before dialysis is greater than that of post dialysis samples [7].

Dielectric property of human blood carries a rich variety of information regarding the functioning of body organs [8]. Achieving a better characterization and understanding of these properties not only is of academic interest but also of high relevance for medical applications such as it helps in correct diagnosis of diseases in human body $[9,10]$.
Similarly the dielectric properties of blood changes with the creatinine content in it.

\section{Methodology}

For the analysis of pre and post dialysis kidney disease patients, blood samples of 25 patients were collected from dialysis centers of GTB hospital, Delhi and care plus pathology laboratories, Ghaziabad. $5 \mathrm{ml}$ of the blood was obtained from each patient before and after dialysis. The blood was placed in clot activator tubes. Clotted blood was centrifuge to separate serum and was used for the estimation of creatinine. The serum was preserved in care plus pathology lab, Ghaziabad as shown in Figure1. Creatinine of each sample has been measured very accurately.

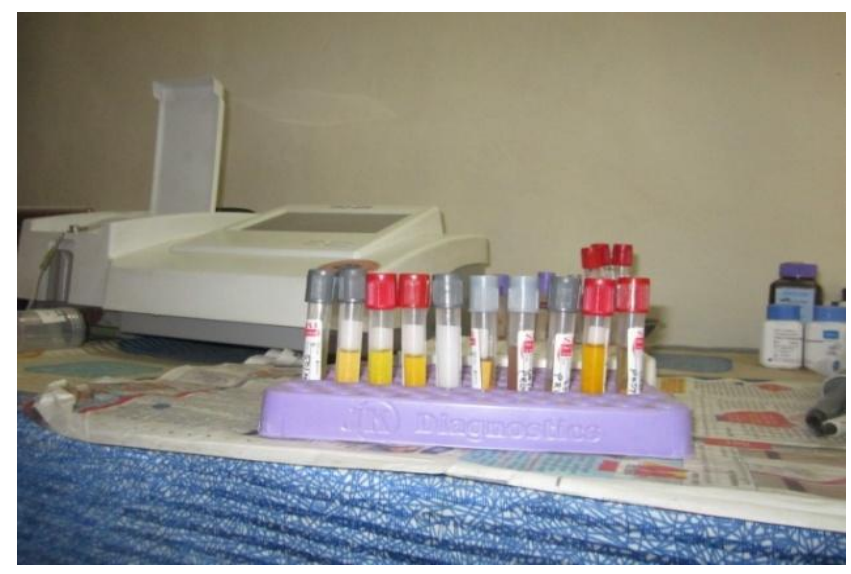

Figure 1: Blood Serum

The dielectric of each samples were measured using DAK as shown in figure 2. DAK is used for measuring dielectric of liquids, solids and semisolids. It is ideal for all applications where high precision dielectric measurements are required. The software of DAK includes advanced graphical visualization and data analysis tools. The interfacing of electrode and software is shown in figure 3. The experimental setup is shown in figure 4 . 


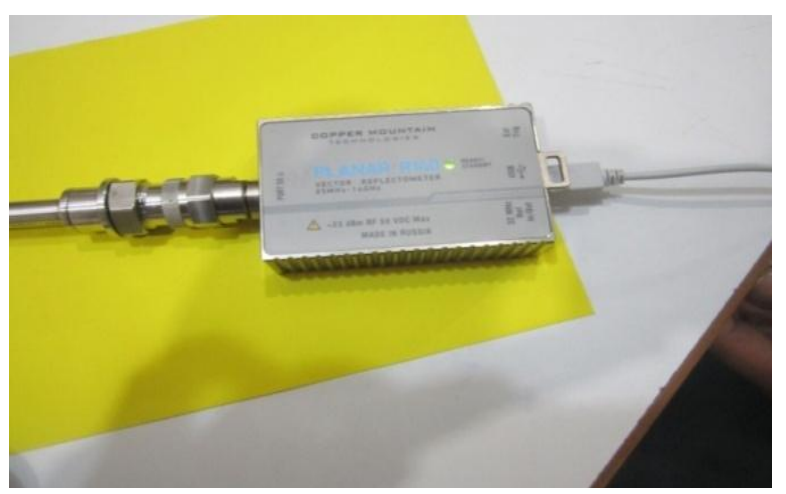

Figure 2: DAK Dielectric Meter

Step1: Using measuring beaker $20 \mathrm{ml}$ deionised water was taken in a separate beaker.

Step2: Using a syringe $5 \mathrm{ml}$ blood serum was taken and stirred well with the deionised water.

Note: The mixture was prepared as to increase the volume of the blood serum in order to carry on the experiment.

Step3: Using DAK the dielectric of each samples were measured keeping temperature and other room conditions constant.

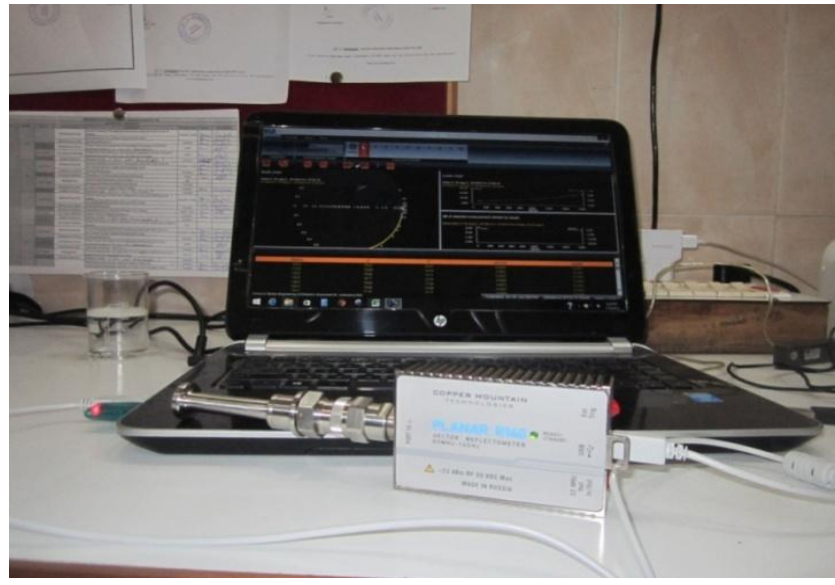

Figure 3: Interfacing the Electrode with software

\section{Result and Observation:}

We observed that the value of creatinine in pre dialysis blood sample is higher than that of post dialysis sample of all the collected blood samples. The creatinine value changes the blood dielectric. The observed value of three patients randomly selected is given in table 1 .

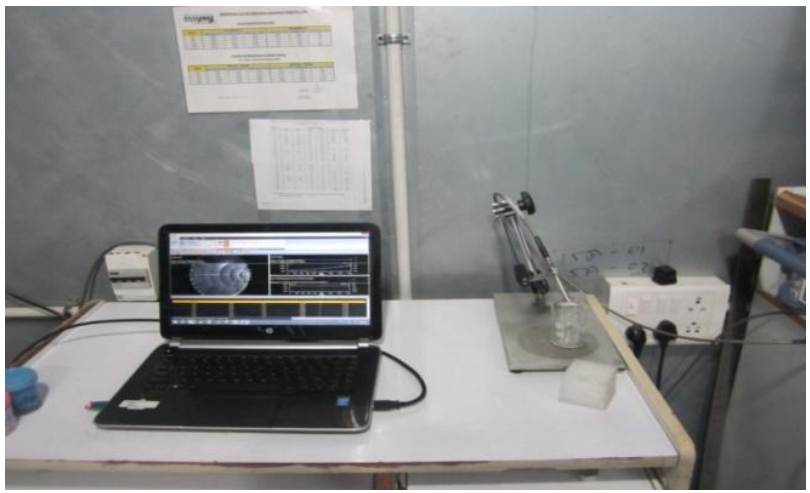

Figure 4: Experimental Setup

Table1: The change in dielectric with creatinin

\begin{tabular}{|c|c|c|c|c|c|c|}
\hline \multirow{2}{*}{$\begin{array}{c}\text { Fequency Range (MHz)// } \\
\text { parameter }\end{array}$} & Dielectric of Patient 1 & \multicolumn{2}{|c|}{ Dielectric of Patient 2 } & \multicolumn{2}{|c|}{ Dielectric of Patient3 } \\
\cline { 2 - 7 } & Pre dialysis & Post dialysis & Pre dialysis & Post dialysis & Pre dialysis & Post dialysis \\
\hline Creatinine (mg/d)) & 9 & 5.4 & 6.3 & 3.85 & 6.79 & 4.6 \\
\hline $1200-1400$ & 77.57 & 78.17 & 76.83 & 77.11 & 77.67 & 77.78 \\
\hline $1410-1600$ & 77.44 & 78.27 & 76.52 & 77.08 & 77.71 & 77.85 \\
\hline $1610-1800$ & 77.46 & 78.31 & 76.69 & 77.19 & 77.90 & 77.99 \\
\hline $1810-2000$ & 77.44 & 78.50 & 77.12 & 77.43 & 78.15 & 78.28 \\
\hline
\end{tabular}

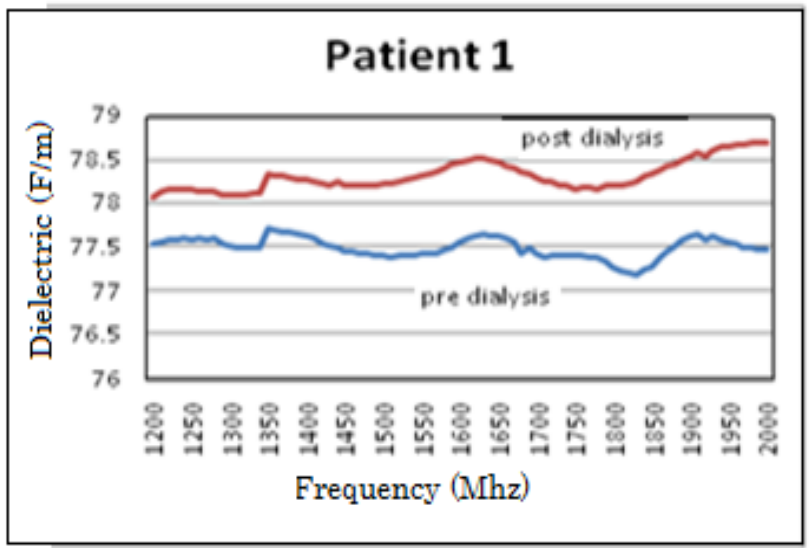

Figure 5 shows the relationship between frequencies on the dielectric change in patient 1 with pre dialysis creatinine value $9 \mathrm{mg} / \mathrm{dl}$ and post dialysis creatinine value $5.4 \mathrm{mg} / \mathrm{dl}$. 
Figure 6 shows the relationship between frequencies on the dielectric change in patient 2 with pre dialysis creatinine value $6.3 \mathrm{mg} / \mathrm{dl}$ and post dialysis creatinine value $3.85 \mathrm{mg} / \mathrm{dl}$.

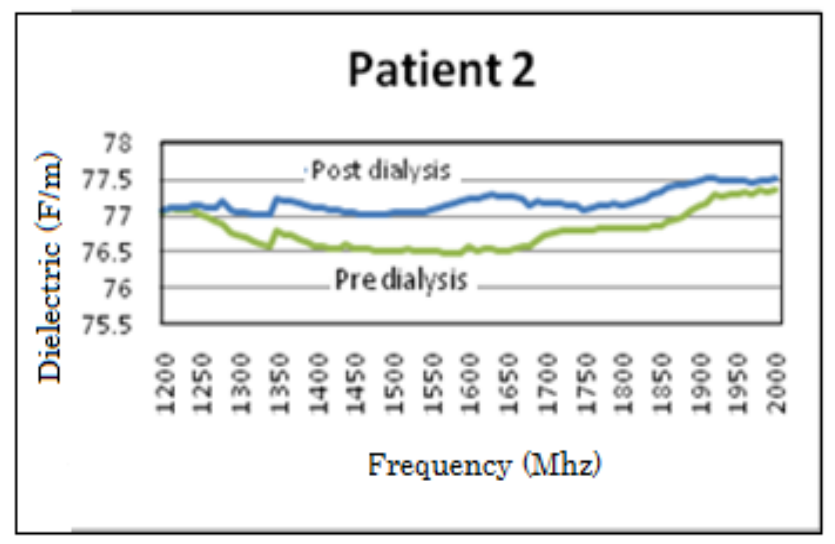

Figure 6: Change in Dielectric with Frequency of Patient 2

Figure 7 shows the relationship between frequencies on the dielectric change in patient 3 with pre dialysis creatinine value $6.79 \mathrm{mg} / \mathrm{dl}$ and post dialysis creatinine value $4.6 \mathrm{mg} / \mathrm{dl}$.

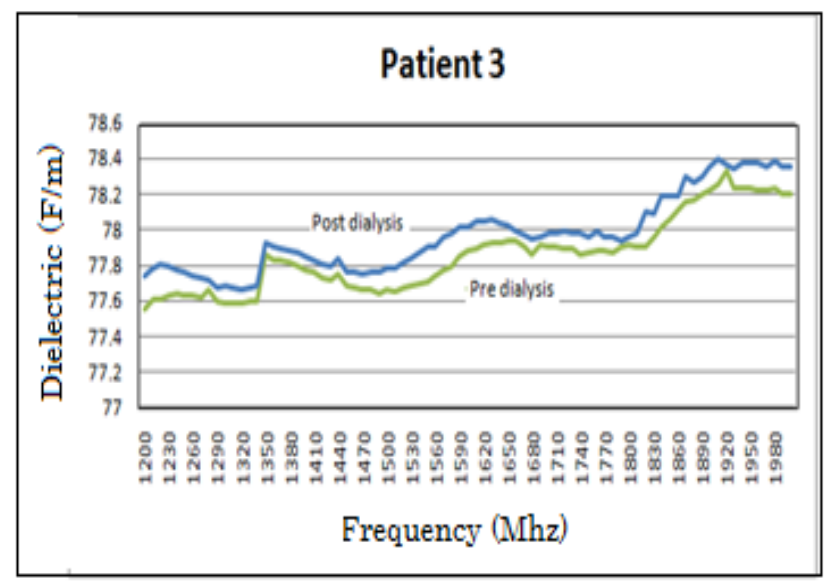

Figure 7: Change in Dielectric with Frequency of Patient 3

As we know the capacitance of a capacitor is directly proportional to dielectric constant and area of the plates of the capacitor, and it is inversely proportional to the distance between the two plates.

$\mathrm{C}=\varepsilon \mathrm{A} / \mathrm{D}$

To study the change in capacitance we used the blood samples as the dielectric of a capacitor where Area and distance was kept constant. A standard conductivity cell was selected for capacitance studies of blood and its constituents. This cell contains two parallel plates, the leads of which plug directly into the live terminals of capacitance measuring bridge.

Dielectric constant $(\varepsilon)=\mathrm{Cs} / \mathrm{Ca}=\left(\mathrm{Cs}^{\prime}-\right.$ CL) / $\left(\mathrm{Ca}^{\prime}-\mathrm{CL}\right)$, where, $\mathrm{Cs}=$ Actual sample capacitance and $\mathrm{CL}=$ Lead capacitance, $\mathrm{Ca}=$ Actual air capacitance and $\mathrm{Cs}^{\prime}=$ Measured capacitance of cell with liquid $\mathrm{Ca}^{\prime}=$ Measured capacitance of cell without liquid, at $1 \mathrm{KHz}$ frequency. Using this relation the capacitance of blood cell has been measured. The observed relation between creatinine and the capacitance is as shown in Figure 8.

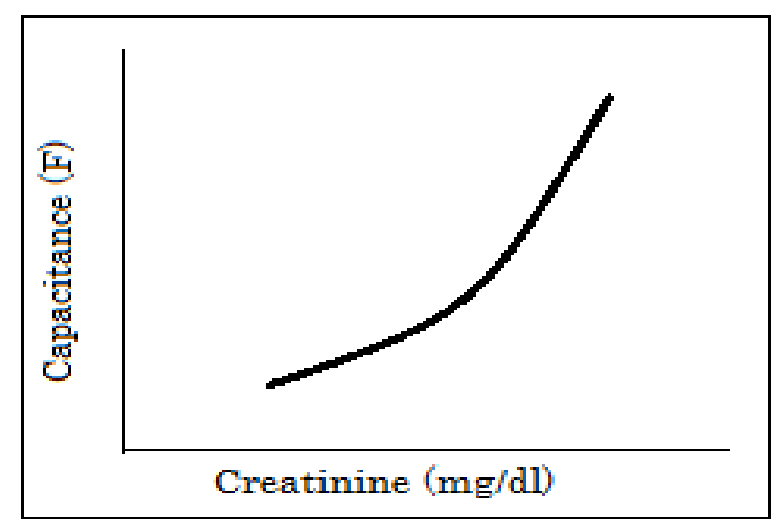

Figure 8: Relation between creatinine and capacitance

\section{Conclusion}

Using MINITAB software we analysed the observation of the experiment.

Table-2 Change in diectric of blood cell with creatinine at different frequencies

\begin{tabular}{|c|c|c|c|}
\hline \multirow{2}{*}{ Parameters } & \multicolumn{3}{|c|}{ Difference of Pre and Post Dialysis Readings } \\
\cline { 2 - 4 } & Patient 1 & Patient 2 & Patient 3 \\
\hline Creatinine $(\mathrm{mg} / \mathrm{dll}) \Rightarrow$ & 3.6 & 2.45 & 2.19 \\
\hline Frequency MHz & & & \\
\hline $1200-1400$ & 0.60 & 0.29 & 0.11 \\
\hline $1410-1600$ & 0.83 & 0.56 & 0.14 \\
\hline $1610-1800$ & 0.85 & 0.51 & 0.09 \\
\hline $1810-2000$ & 1.05 & 0.32 & 0.13 \\
\hline
\end{tabular}


Table-1 shows summarized results of dielectric in different frequency range. From the table- 2 it is concluded that

1) The difference (pre and post dialysis) of absolute values of Creatinine and dielectric are directly proportional to each other.

2) Value of Creatinine decreases after the dialysis while Value of dielectric increases.

3) Stability in results of dielectric has been observed.

4) The results after regression analysis shows that the $\mathrm{R} \mathrm{sq}$ value for all 4 set of frequencies is greater than $78 \%$ and the $\mathrm{R}$ value $>80 \%$ indicates that the model fits the data extremely well. MINITAB result is shown in figure 9.

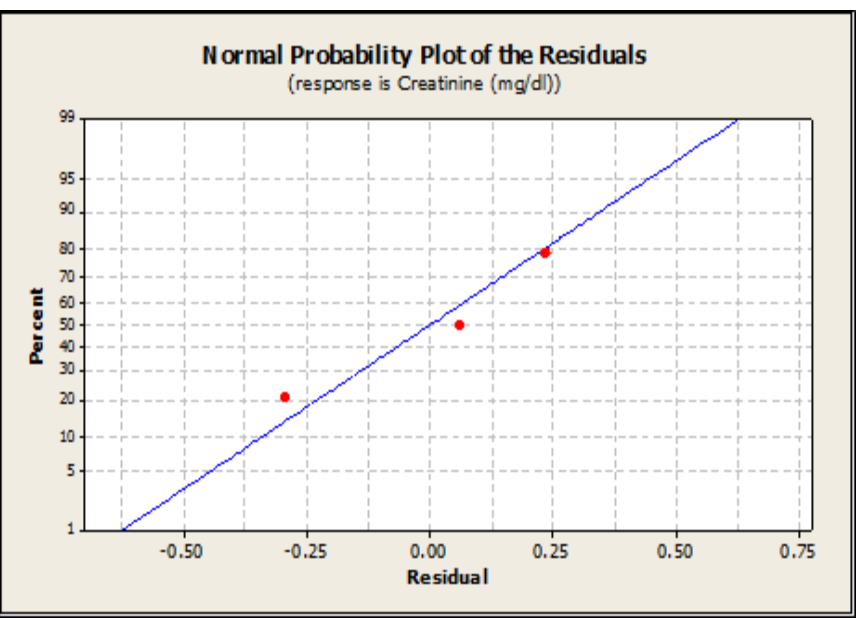

Figure 9: MINITAB Result

\section{Acknowledgement}

The authors are thankful to the management of Krishna Engineering College, Ghaziabad for providing us the necessary support to carry out the experiments. We are grateful to the Mr Nitin Jain of BNN Communication, Ghaziabad for helping and guiding us. We thank the doctors and staff of GTB hospital, Delhi and Care Plus pathology Labs. We also thank $\mathrm{Mr}$ Ravindra Gupta Asst General Manager-Quality in Precision Electronics Ltd, Noida for his guidance in analyzing the data statistically using MINITAB software. We extend our sincere thanks to Mrs Warrior.

\section{References}

1) Noor ul amin, Raja Tahir Mahmood, M. Javaid Asad, Mudassar Zafar and Asad Mehmood Raja, Evaluating urea and creatinine levels in chronic renal failure pre and post dialysis: a prospective study, journal of cardiovascular disease, (2014) vol.2 no.2, ISSN: 2330-4596 (print) / 2330460x (online).

2) Levey A S, Atkins R, Coresh J, Cohen E P, Collins A J and Eckardt K U Chronic kidney disease as a global public health problem: Approaches and initiatives -a position statement from Kidney Disease Improving Global Outcomes. Kidney International; (2007) 72: 247-259. DOI: 10.1038/sj.ki.5002343

3) Levey A S, Coresh J, Balk E, Kausz A T, Levin A and Steffes M W. National Kidney Foundation practice guidelines for chronic kidney disease: evaluation, classification, and stratification Annals of Internal Medicine; (2003) 139(2): 137-47 3.

4) Coresh J, Selvin E, Stevens L A, Manzi J, Kusek J W and Eggers P. Prevalence of chronic kidney disease in the United States Journal of the American Medical Association, (2007), 298(17): 2038- 47

5) Couchoud C, Pozet $N$ and Labeeuw $M$. Screening early renal failure: cut-off values for serum creatinine as an indicator of renal impairment. Kidney International, (1999) 55: 1878-1884 DOI: 10.1046/j.15231755.1999.00411.X

6) Hayrullah Y, Mehmet B, Mustafa B K, Yesim G A and Sadik B. The effects of dialysers on some blood biochemical parameters in hemodialysis patients. Africian Journal of Pharmacy and Pharmacology, (2011), 5(22): 2513-2516 22.

7) Kaysen G A, Tom G, Larive B and Ravindra L. The Effect of Frequent Hemodialysis on Nutrition and Body Composition: Frequent Hemodialysis Network Trial. Kidney International Journal, (2012), 82(1): 90-99 DOI: $\underline{10.1038 / \mathrm{ki} .2012 .75}$ 
8) T.K.Basak, Arpita Gupta, G.U.Kharat, Vivek Srivastava, Poonam Goyal.The Disease Dependent Electrical Properties of Living Cell -A Review, proceedings of international conference ICCE held in KIET Ghaziabad, (2012), (pp 180-184)

9) Akihiko Irimajirit, Shinobu S Uzaki.K. Ji Asami.A.N Tetsuya Hanai, Dielectric Modeling of Biological Cells. Models and Algorithm, Bull. Inst. Chem. Res., Kyoto Univ, (1991) Vol. 69, No. 4, (421-428)
10) Arpita Gupta, G.U.Kharat Modeling Of Dielectric Properties Of Cancer Cell And Evaluation Of Cancer Stages: A Review, International Journal of Recent Scientific Research (2014) Vol. 5, Issue, 2, Pp.443-448 